\title{
OPTIMIZATION OF THE THERMAL STATE OF THE CYLINDER SLEEVE OF THE MEDIUM-ROTATION MARINE DIESEL ENGINE
}

\section{A.V.Trinev, R.Yu.Buhaitsov}

The calculation study is based on the results of long-term experimental studies of the thermal state of a cylindrical sleeve of the middle-speed marine diesel 6CHN26 / 34, which were carried out at the Department of DVS NTU "KhPI" in the 80s under the guidance of prof. Ye. I. Tretyak. The problem of marine diesel engines of this design was a significant supercooling of the liner, which, in turn, worsened the lubrication conditions in the liner-piston coupling, due to the increased viscosity of the engine oil. Optimization of the thermal state provided for the achievement of a certain temperature profile along the working surface of the liner, such that it is possible to minimize friction costs while maintaining the viscosity of the oil within certain limits. The carried out experimental studies confirmed the possibility of practical solution of this problem.

In order to improve the technical and economic parameters of the ship's diesel engine, this design study sets the task of developing a mathematical model for the heat-stressed state of a cylindrical sleeve, its refinement on the basis of these experimental studies, and the development of practical recommendations for optimizing the temperature condition of the sleeve.

The analysis of publications on the research subjects, in particular, the works of prof. E.I. Tretyak, the features of specifying the boundary conditions of the heat conduction problem for design options are analyzed in detail. In particular, it has been proposed to limit the cooling of the liner only by local cooling of its upper belt. To equalize the temperatures along the height of the sleeve, it is also proposed to apply a heat-insulating enamel layer on the lower part of the sleeve. The heat-stressed state of the sleeve is analyzed using the finite element method.

\section{А.Ю. Яковенко}

\section{МЕТРОЛОГИЧЕСКИЕ ХАРАКТЕРИСТИКИ РЕГИСТРАЦИИ ВЫСОКОГО ДАВЛЕНИЯ В СИСТЕМАХ ТОПЛИВОПОДАЧИ ДИЗЕЛЕЙ С ПРИМЕНЕНИЕМ КОМБИНИРОВАННОГО ДАТЧИКА}

\begin{abstract}
Представлена методика гармонического анализа метрологических характеристик записи нестационарных прочессов. Использован метод Фурье в приложении к задачам обработки данных, в графической и табличной форме. Коэффициенты Фурье вычислены при помощии приближенного метода интегрирования с использованием формул прямоугольников. Разработан алгоритм и программа, адаптированные к измерению параметров топливоподачи дизелей. Программная реализачия имеет широкий спектр опиий, обеспечивающий оптимальный интерфейс и форму представления результатов анализа. Проведено исследование амплитудно-частотных параметров измерительной системы на базе комбинированного преобразователя давления топлива. В качестве преобразователя использован конструктивный элемент топливной аппаратуры судового среднеоборотного дизеля. Давление топлива в ТНВД записывалось двумя датчиками: базовым традиционной конструкции и опытным. Проведены безмоторные испытания в диапазоне частот вращения, перекрывающих эксплуатационные режимы двигателя. Экспериментальные данные обработаны в среде разработанного пакета. Определены амплитудно-частотные характеристики измерительной системы в сравнении комбинированного и базового преобразователя давления топлива. Произведено дискретное преобразование Фурье с выводом результата в виде графика амплитуд спектральных составляющих на экран и для визуального контроля обратное преобразование Фурье также с выводом графика функиии на экран. Установлено, что система с комбинированным преобразователем имеет параметры близкие к базовому варианту на всех режимах за исключением дробного впрыскивания.
\end{abstract}

\section{Введение}

Одним из основных процессов, определяющих все эксплуатационные характеристики дизелей, является впрыскивание топлива. В области его мониторинга остаются нерешённые задачи, связанные с высокой динамичностью процессов, их периодичностью и метрологической сложностью контроля топливоподачи.

Основным параметром, определяющим качество регистрации динамических процессов, является частотная характеристика.

Для её расчёта строится гармонический ряд, аппроксимирующий кривую процесса. Наиболее употребительный инструмент частотного анализа метод Фурье.

Амплитудно-частотный анализ записи рабочих процессов дизелей измерительными системами различной конфигурации позволяет дать оценку достоверности информации и обеспечить корректировку полученных данных при наличии погрешностей.

Развитие средств диагностики двигателей внутреннего сгорания идёт в направлении контроля рабочего процесса по индикаторной диаграмме [13]. При записи индикаторной диаграммы оптимальным было бы расположение датчика давления заподлицо со стенкой камеры сгорания, но в усло- 
виях эксплуатации судовых дизелей это условие трудновыполнимо. В настоящее время индицирование мало- и среднеоборотных дизелей осуществляется через индикаторный канал с краном. В канале индикаторного крана возникают волновые явления, которые искажают индикаторную диаграмму.

Для коррекции одноцикловой индикаторной диаграммы используется [4] метод цифровой фильтрации на основе прямого преобразования Фурье. В полученном спектре выделяется диапазон значимых частот, а амплитуды остальных частот приравнивались к нулю. Для восстановления сигнала давления из спектра выполнялось обратное преобразование Фурье.

\section{Цели статьи}

В данной работе ставилась задача создания алгоритма и программы, обеспечивающих обработку опытных данных, представленных в виде осциллограмм, а также исследования метрологических характеристик системы на базе комбинированного преобразователя давления топлива в насосе высокого давления (ТНВД) [1].

Изложение основного материала исследования

В общем случае задачей гармонического анализа является представление сложного негармонического колебания в виде суммы гармонических колебаний, образующих, так называемый спектр колебания. Если сложное колебание - периодическая функция с частотой $v=1 / T$ и с периодом $T$, то его спектр дискретный, или линейчатый и он состоит из гармонических колебаний с частотами, кратными v.

Если анализируемая функция является периодической функцией $x(t)$ с периодом $T$, то ее можно представить в виде

$$
x(t)=a_{0}+\sum_{k=1}^{\infty}\left(a_{k} \cos \left(k \omega_{1} t\right)+b_{k} \sin \left(k \omega_{1} t\right)\right)
$$

либо

$$
x(t)=c_{0}+\sum_{k=1}^{\infty} c_{k} \cos \left(k \omega_{1} t-\varphi_{k}\right),
$$

где $c_{k}=\sqrt{a_{k}^{2}+b_{k}^{2}}, \operatorname{tg} \varphi_{k}=\frac{a_{k}^{2}}{b_{k}^{2}}, \omega_{1}=\frac{2 \pi}{T}, t$ время, $T$ - период, $k$ - номер гармоники, $a_{k}, b_{k}, c_{k}-$ коэффициенты Фурье, $a_{0}, c_{0}-$ постоянные, $\omega_{1}-$ круговая частота; $\varphi_{\mathrm{k}}$ - фаза $k$-ой гармоники.

Зависимость значений амплитуд $c_{k}$ от частоты или от номера гармоники является амплитудным спектром функции, а зависимость значений начальных фаз $\varphi_{k}$ от частоты или от номера гармоники называется фазовым спектром функции.
Во многих случаях, встречающихся на практике, исходная функция $f(x)$ задается или в виде таблицы или в виде графической кривой. В этих случаях применяется приближенное представление периодической функции $f(x)$ тригонометрическими многочленами вида,

$$
s_{n}=a_{0}+\sum_{k=1}^{n}\left(a_{k} \cos (k x)+b_{k} \sin (k x)\right)
$$

а коэффициенты Фурье вычисляются при помощи приближенных методов интегрирования, например, с использованием формул прямоугольников

$$
\begin{aligned}
& a_{0}=\frac{1}{n} \sum_{k=1}^{n} y_{k} ; \quad a_{k}=\frac{2}{n} \sum_{k=1}^{n} y_{k} \cos \left(k x_{k}\right) ; \\
& b_{k}=\frac{2}{n} \sum_{k=1}^{n} y_{k} \sin \left(k x_{k ;}\right) ; \quad c_{k}=\sqrt{{a_{k}{ }^{2}+b_{k}^{2}}^{2} .}
\end{aligned}
$$

Преимущество приближенного представления функции, таким образом, заключается в том, что тригонометрический ряд, коэффициенты которого суть коэффициенты Фурье, имеет наименьшее среднеквадратичное отклонение от представимой функции $f(x)$ среди всех тригонометрических многочленов порядка $n$.

Для анализа частотных параметров данных, связанных с разработкой комбинированного датчика давления топлива с помощью рядов Фурье, была составлена программа для персонального компьютера (ПК).

Давление топлива в ТНВД записывалось двумя датчиками: базовым традиционной конструкции и опытным. Последний был совмещён со шпилькой крепления ТНВД к двигателю.

Общий вид комбинированного датчика «шпилька-датчик» (Ш-Д), подготовленного к испытаниям, дан на фотографии рис. 1. На фотографии рис. 2 показан ТНВД с датчиком «Ш-Д» на безмоторном стенде.

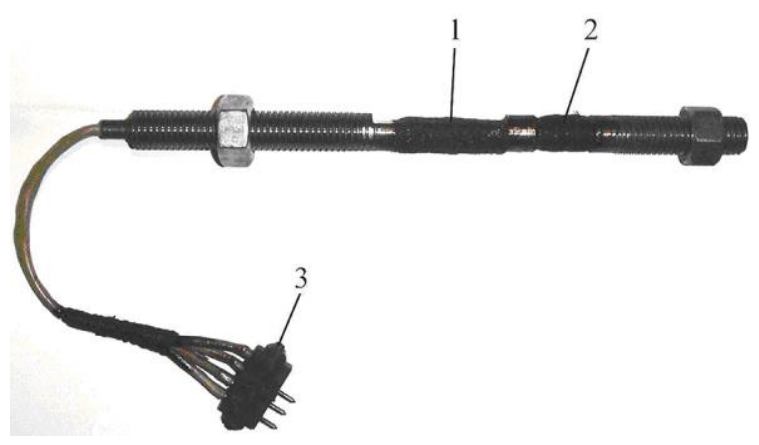

Рис. 1. Препарированная шпилька с датчиком «Ш-Д»: 1- активные датчики, 2 - компенсационные датчики, 3 - электрический разъём 
Алгоритм пакета предусматривает выполнение спектрального анализа периодических процессов, представленных в виде дискретного ряда с полной длиной не более 20000 точек. Результат работы программы - график амплитуд гармонических составляющих, причем число анализируемых гармоник устанавливается пользователем в диапазоне 1500 .

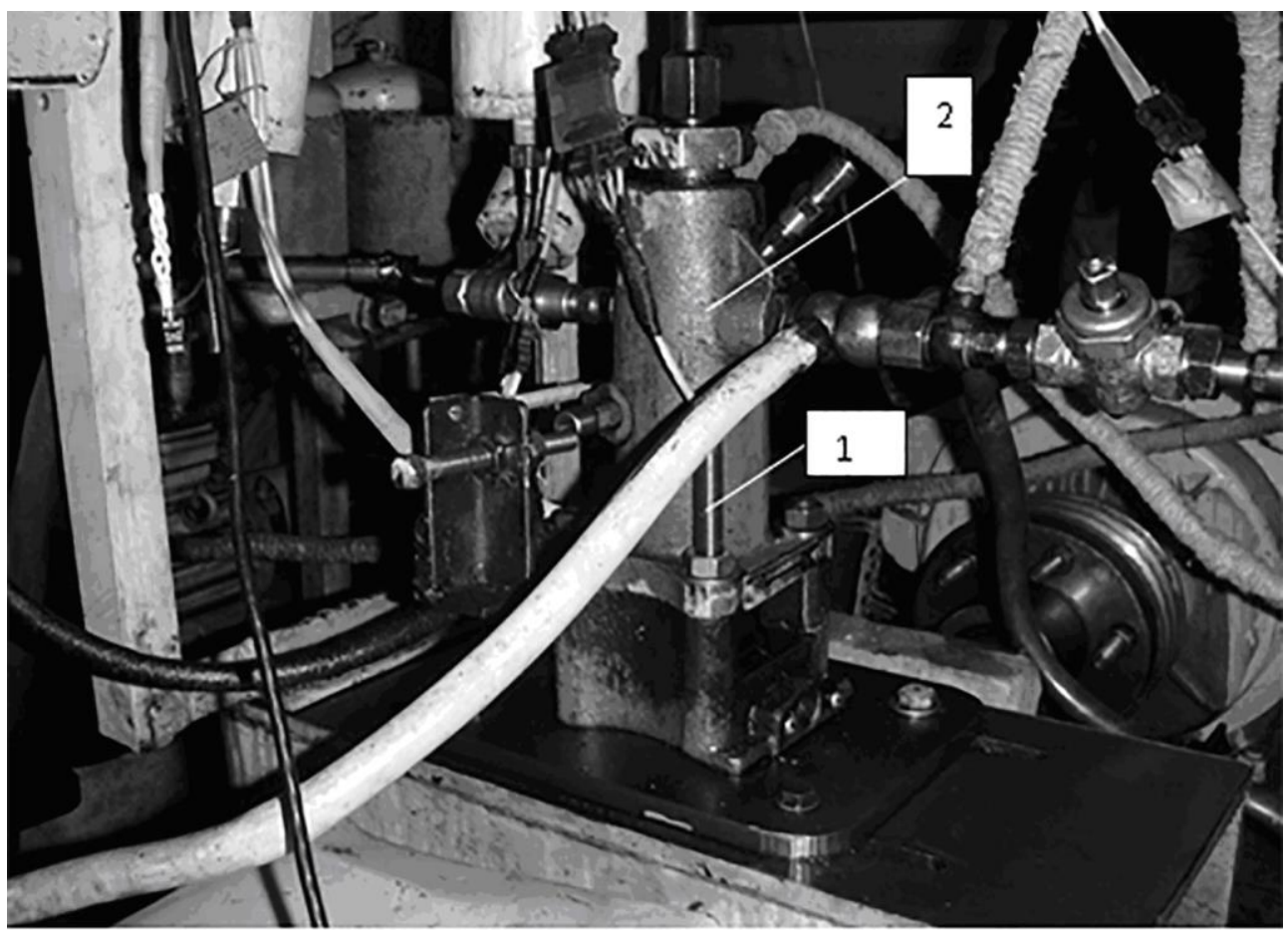

Рис. 2. Датчик давления топлива «Ш-Д» на ТНВД безмоторного стенда: 1 - шпилька с датчиком «Ш-Д», 2 - ТНВД

Исходный файл данных - это дискретный ряд чисел, представляющий собой часть периода анализируемого процесса. Для дальнейшего анализа исходный ряд преобразуется таким образом, чтобы представлять один полный период процесса. Для этого после загрузки файла данных в диалоговом режиме вводятся следующие исходные параметры:

- частота вращения распределительного вала, $\mathrm{Mин}^{-1}$;

- начальная фаза процесса для первой точки исходного ряда, в градусах поворота распределительного вала $\left({ }^{0} \Pi К В\right)$;

- конечная фаза процесса для конечной точки исходного ряда, ${ }^{0} П К В$;

- минимальное и максимальное давления топлива, МПа.

После ввода указанных параметров производится расчет новых данных уже для полного периода процесса с выводом результата в виде графика на экран.

Далее производится дискретное преобразование Фурье (4) с выводом результата в виде графика амплитуд спектральных составляющих на экран и для визуального контроля обратное преобразование Фурье (3) также с выводом графика функции на экран.

После ввода указанных параметров производится расчет новых данных уже для полного периода процесса с выводом результата в виде графика на экран.

Блок-схема пакета приведена на рис.3, а. Кроме собственно расчётной части в ней предусмотрен графический ввод и преобразование в числовую форму осциллограмм. Разработан также графический интерфейс управления программой и графический вывод результатов анализа. Форма представления результатов также устанавливается средствами графического интерфейса.

Пример графического представления результатов амплитудно-частотного анализа представлен на рис. 3,6 .

Выбор рисунка производится двойным щелчком левой кнопки мышки в поле окна требуемого графика, отмена выбора осуществляется также двойным щелчком левой кнопки мышки. 


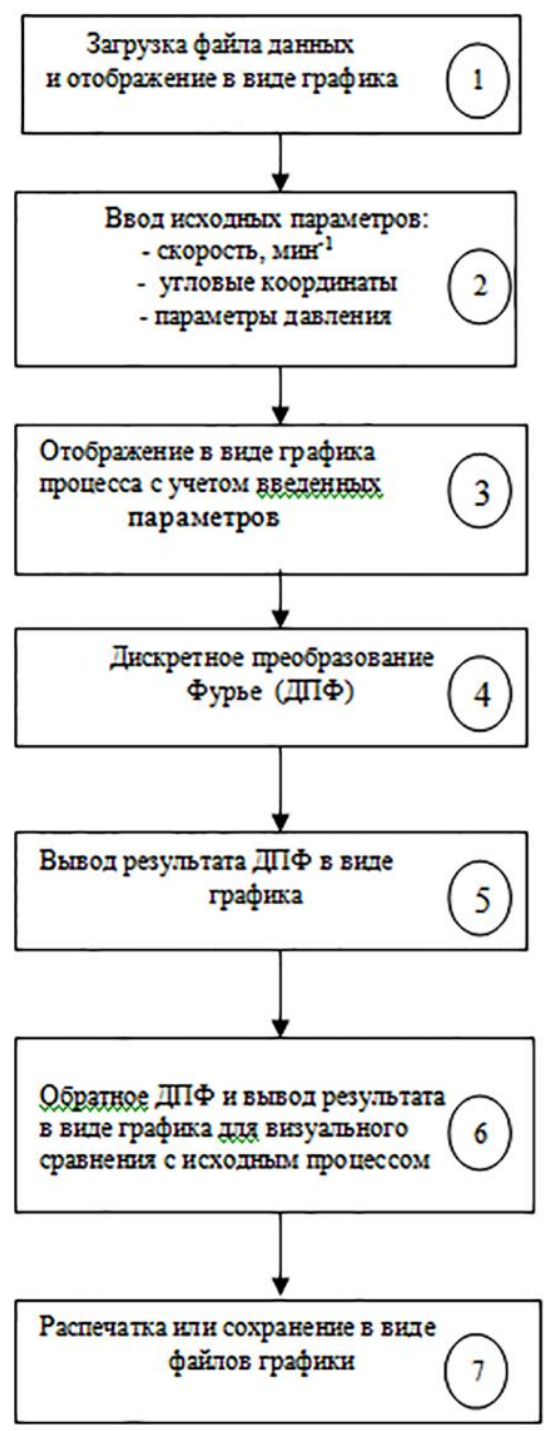

a)

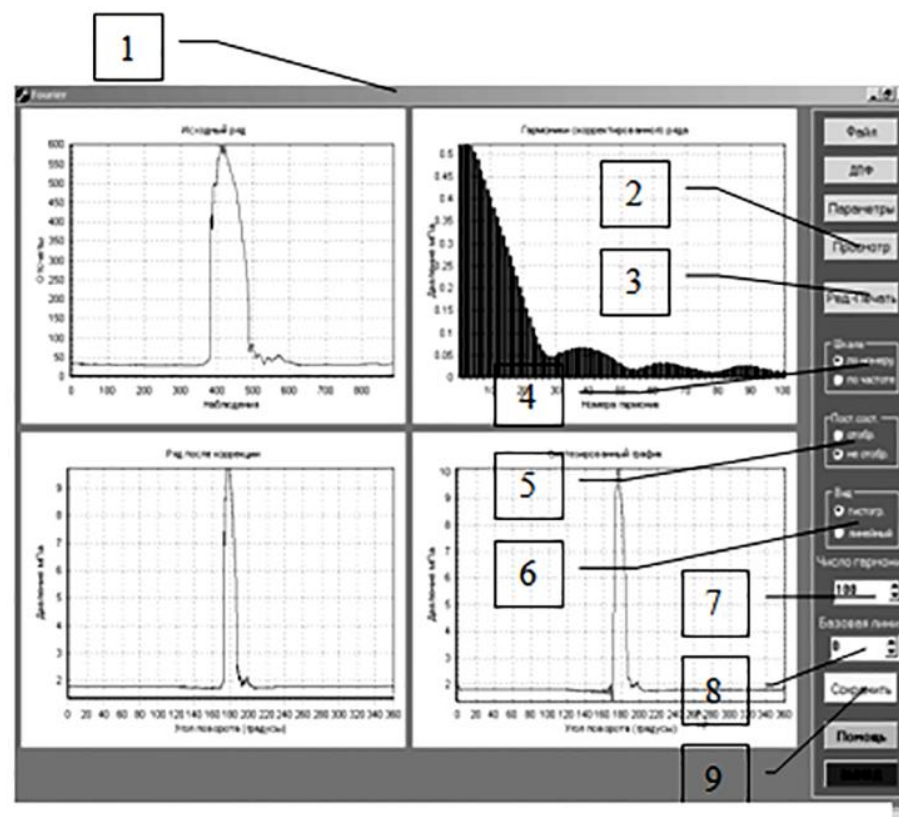

1- вид на экран монитора после нажатия кнопки 'ДПФ'; 2- кнопка 'Просмотр', вызывает на экран окно для просмотра числовых значеннй скорректированного ряда и гармоник; 3- кнопка 'Ред - Печать', вызывает диалоговое окно для выбора графика для редактировання и печати или для записи файла гармоник; 4- переключатель вида отображения для шкалы абсцисс гармоник; 5- переключатель выбора отображения постоянной составляющей; 6переключатель вида отображения графика гармоник; 7- окно ввода числа рассчитьгаемых гармоннк (по умолчанию 100); 8- окно ввода постоянной составляющей; 9- кнопка 'Сохранить', позволяет сохранить выбранный график в электронном виде как файл рисунка формата *.bmp.

б)

Рис.3. Блок-схема и графический интерфейс метрологических характеристик системь на базе комбинированного датчика «Ш-Д»

Программа позволяет оперативно менять число вычисляемых гармоник, вид графика отображения спектра, представление спектра в виде зависимости от частоты либо по номерам гармоник, отображать или нет постоянную составляющую, а также выводить на печать или сохранять в виде электронной копии (рисунок формата ВМР) с возможностью редактирования любого графика.

Приоритетной задачей проведенного исследования с использованием частотного анализа является сопоставление амплитудно-частотных характеристик записи давления топлива двумя типами датчиков при равнозначных параметрах остальных элементов измерительной схемы.

Запись давления топлива проведена синхрон- но двумя датчиками: базовым $p_{\text {н }}$ и комбинированным $p_{ш}$ на базе шпильки крепления ТНВД к двигателю. Пример осциллограммы приведен на рис. 4.

Амплитудно-частотные параметры в записи $p_{\text {н }}$ и $p_{ш}$ имеют некоторые различия. По амплитудам гармоник в области минимальных частот $p_{ш}$ несколько выше. Зона начального массива, наоборот, уже. Так, низкочастотная полоса у $p_{\text {ш }}$ ограничена минимумом номеров гармоник, соответствующих порядку 28, а $p_{\mathrm{H}}-34$.

Для более высоких порядков форма и амплитудные величины для обоих вариантов записи близки. Граничные значения периодических зависимостей смещены в большую сторону (на 3-5 порядков) у $p_{\mathrm{H}}$. 


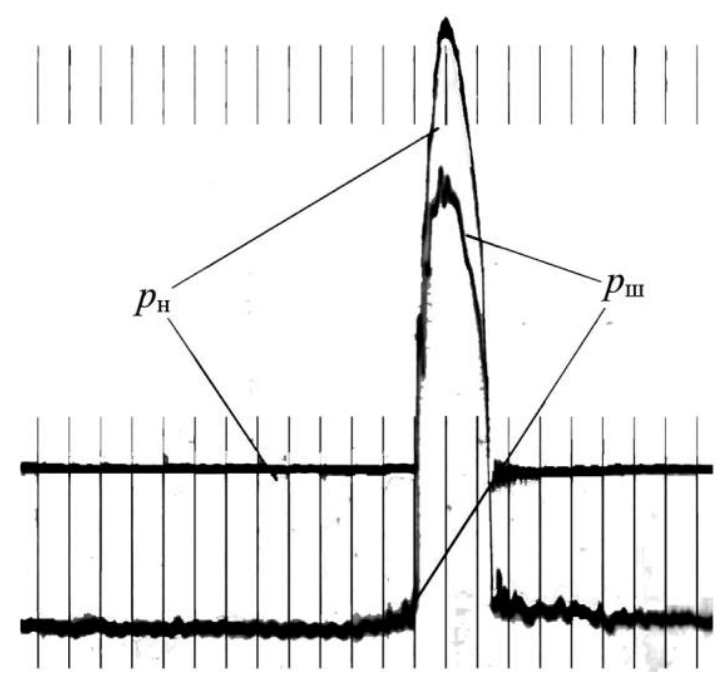

Рис. 4. Осциллограмма топливоподачи: $p_{н}-$ базовылй датчик; $p_{u}-$ комбинированный «Ш-Д» датчик

Различная форма исходных кривых дробного впрыскивания отражена в данных гармонического анализа. При небольшой разнице в максимальных значениях амплитуд распределение их по частотам

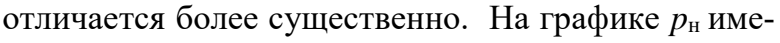
ется непрерывная область вплоть до 84 гармоники, у $p_{ш}$ в диапазоне 20-84 выделаются три отдельные участка в форме затухающих колебаний с перио- дом в 20 порядков.

Разработанные программные средства гармонического анализа позволяют провести сравнение частотных характеристик в графической форме. Такое представление информации приведено на рис. 5,6,7.

Были рассмотрены режимы минимальной частоты вращения $n=33$ и 46 мин $^{-1}$, а также режим $n=248$ мин $^{-1}$, близкий к номинальной частоте вращения распределительного вала.

На каждом графике показаны три кривые. Две относятся к огибающим амплитуд частот для различных датчиков ( $p_{\mathrm{H}}$ и $\left.p_{\text {ш }}\right)$, а третья соответствует их разности $-\left(p_{\text {н }}-p_{ш}\right)$.

Наиболее существенным является различие в параметрах на частоте вращения, равной 33 мин $^{-1}$ (рис.5). Максимальное расхождение относится к 2 Гц, что соотвествует гармонике четвертого порядка. Начиная с частоты $f=10$ Гц, амплитудные значения гармоник обоих датчиков отличаются незначительно.

С повышением частоты вращения частотные параметры датчиков сближаются.

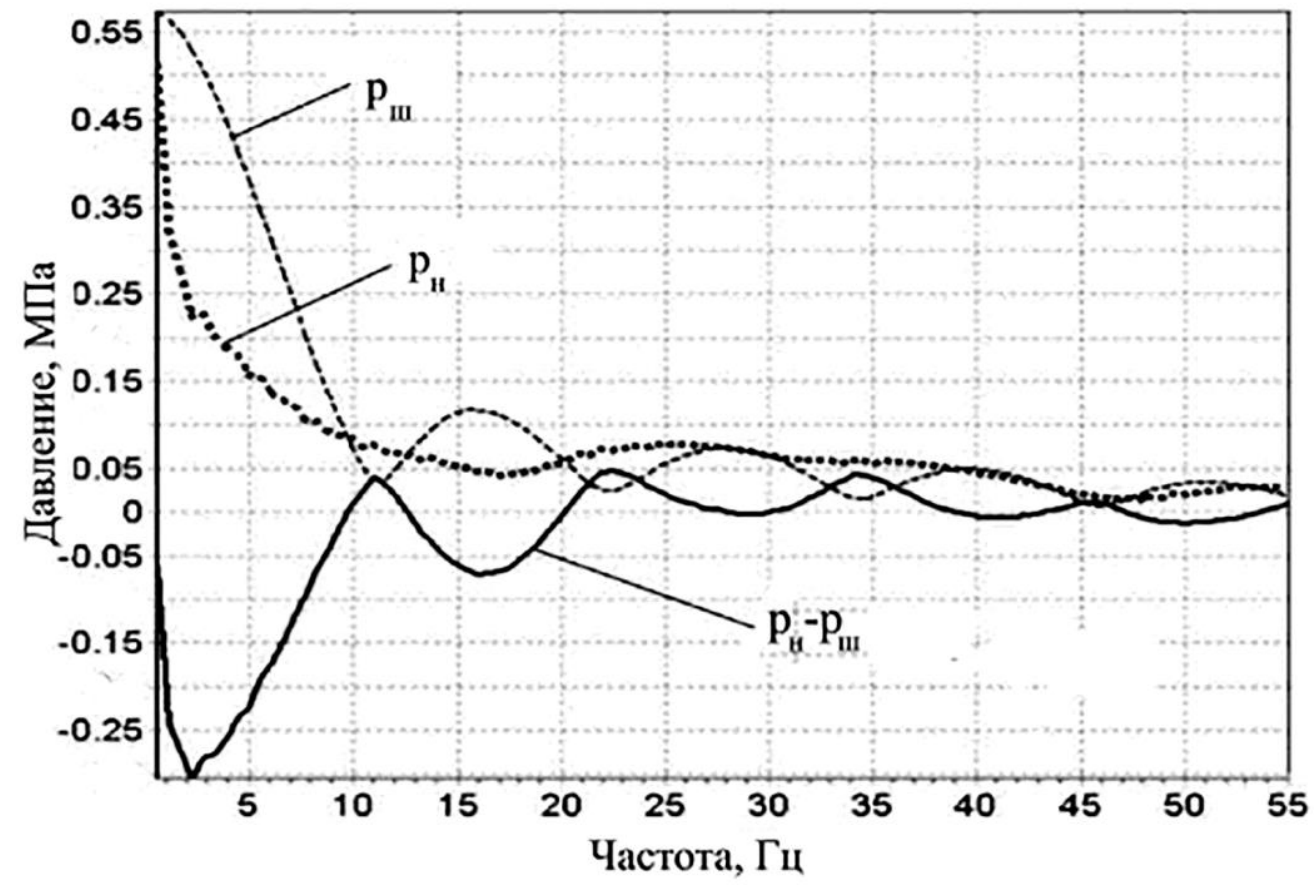

Рис. 5. Сравнение частотных характеристик $p_{н}$ и $p_{u}$ при частоте вращения $n=33$ мин $^{-1}$

На следующей частоте вращения $n=46$ мин $^{-1}$ (рис. 6) значительного различия нет (разность амплитуд почти в 5 раз меньше по сравнению с предыдущим режимом). Что касается частотного 
диапазона рассматриваемых гармоник, то он расширяется с ростом частот ы вращения.
Так, если в первом случае он укладывается в 10 Гц, то при $n=46$ мин $^{-1}$ это уже 20 Гц.

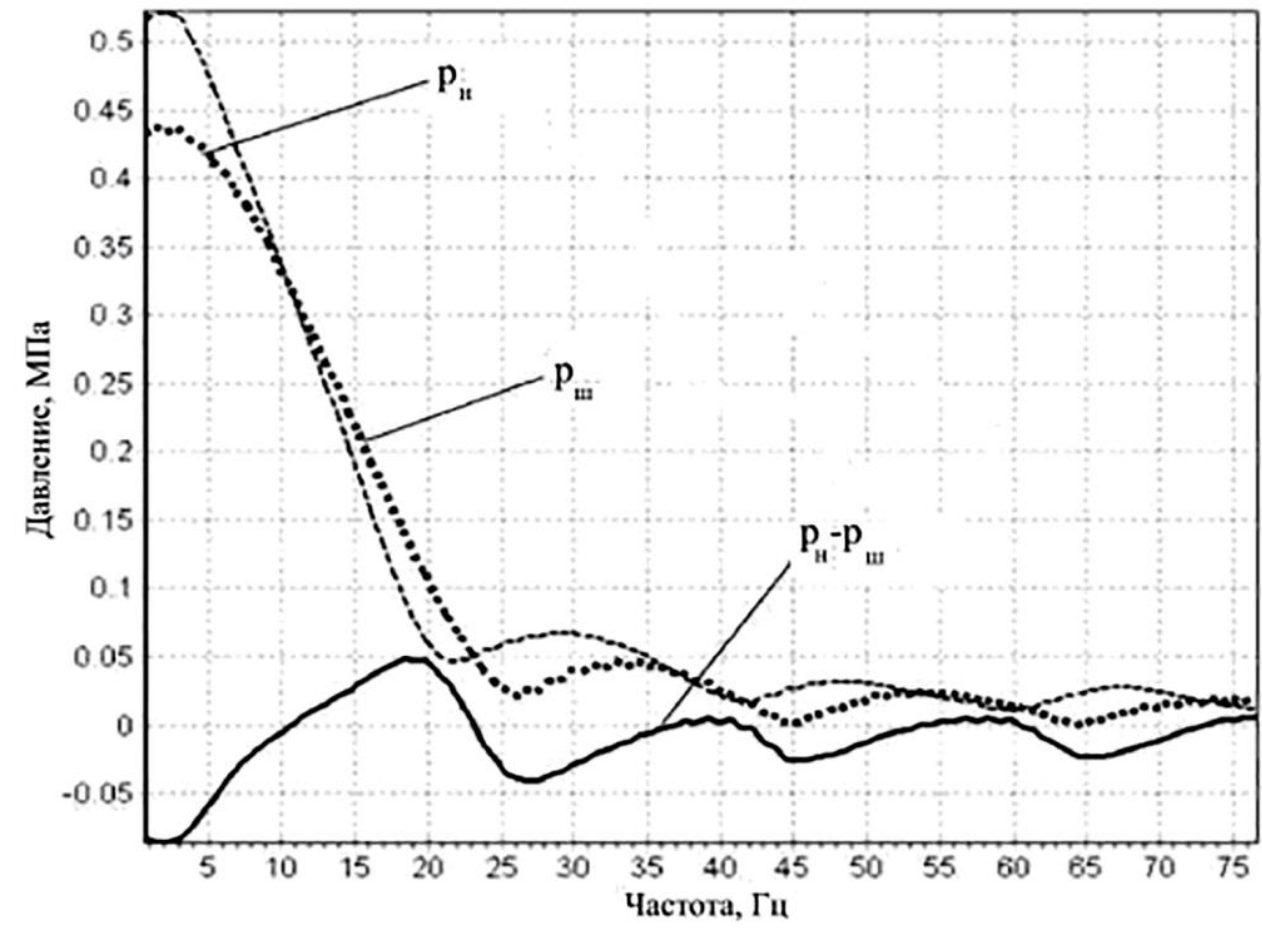

Рис. 6. Сравнение частотных характеристик $p_{н}$ и $p_{u} n р и n=46 м н^{-1}$

Качественно эта тенденция справедлива и на третьем из рассматриваемых режимов $n=248$ мин $^{-}$ 1 (рис. 7). Кривые $p_{\text {н }}$ и $p_{\text {ш }}$ отличаются незначительно, изменяется лишь знак разности. Диапазон существенных частот продолжает расширяться и составляет 70 Гц.

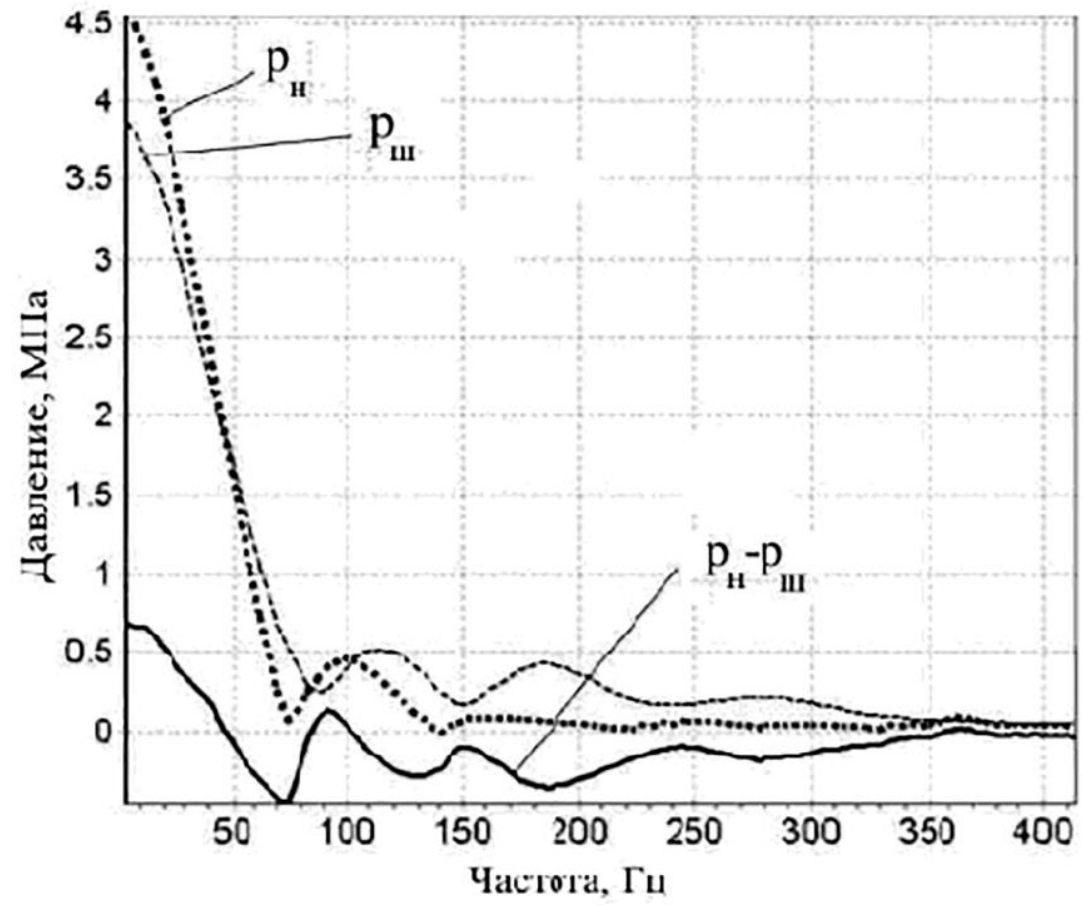

Рис. 7. Сравнение частотных характеристик $p_{н}$ и $p_{u}$ при $n=248$ мин $^{-1}$

Для анализа метрологических особенностей записи давления с помощью датчика «Ш-Д» необ- ходимо провести оценку влияния собственных колебаний элементов, участвующих в передаче уси- 
лия от давления топлива в ТНВД к датчику «Ш-Д». Логичным является вызвать эти колебания независимым источником - с помощью ударной нагрузки.
Такой эксперимент был проведен, а запись деформаций датчика «Ш-Д» представлена на рис.8.

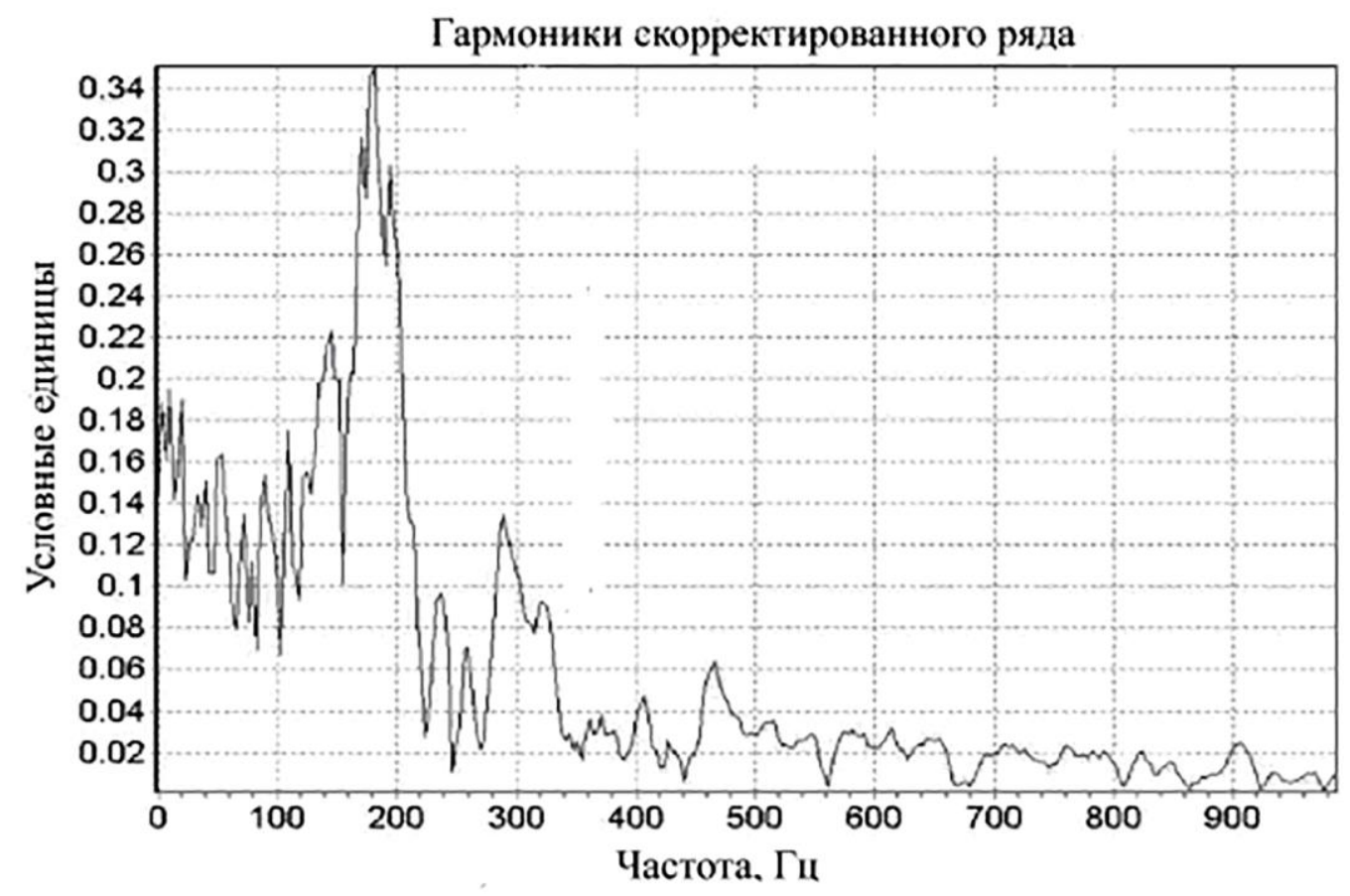

Рис. 8. Частотные характеристики записи собственных колебаний стенда

Частотный диапазон для анализа установлен со значительным запасом по отношению к режиму максимальной частоты вращения (превышение более чем в два раза). Шкала амплитуд является условной, поскольку сопоставление выполняется по суммарному значению в условиях процесса впрыскивания.

Сравнивая запись $p_{\text {ш }}$ на различных режимах впрыскивания с частотной характеристикой собственных колебаний, остановимся, в первую очередь, на диапазонах, где значения амплитуд гармоник являются значительными. Как отмечено выше это частоты с верхними значениями $f=10$, 20 и 70 Гц.

Обращаясь к графику собственных колебаний отметим что, очевидно, запись на рис. 5 - 7 является суммарной от действия давления топлива и влияния собственных колебаний. Характер последних ясен из рис. 8. В рабочей записи не просматривается их влияние, что свидетельствует о незначительной амплитуде гармоник собственных колебаний.

Что касается более высоких частот, то кроме их незначительного участия в формировании рабо-

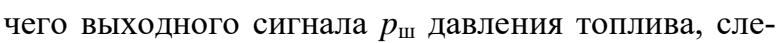
дует отметить отсутствие видимого влияния собственных колебаний измерительной системы. Это справедливо, в том числе, и для участка с макси- мальными амплитудами собственных колебаний $f \approx 150-200$ Гц.

\section{Выводы}

Представленная в статье методика гармонического анализа и программный пакет обеспечивают специализированный подход в определении метрологических характеристик измерительных систем нестационарных процессов. Исходные данные представляются в естественной форме - осциллограммами.

Проведено исследование амплитудночастотных параметров измерительной системы с базовым и экспериментальным датчиками давления в системе топливоподачи среднеоборотного судового дизеля. В качестве экспериментального использован комбинированный преобразователь, совмещённый с конструктивным элементом привода топливного насоса высокого давления.

Амплитудно-частотные характеристики экспериментального преобразователя обеспечивают близкое к базовому датчику качество регистрации давления топлива в ТНВД на режимах стабильного впрыскивания.

При дробном впрыскивании наблюдается демпфирование колебаний, наиболее заметное для четвёртого порядка. 


\section{Список литературы:}

1. Коньков А.Ю. Диагностирование дизеля на основе идентификации рабочих прочессов [Текст]: моногр. / А.Ю. Коньков, В.А. Лашко. - Владивосток: Дальнаука, 2014. - 356 c. 2. Определение основных параметров рабочего прочесса и результаты диагностики главных дизелей теплохода «GREISFSWALD» [Tекст]/ P.A. Варбанеи, П.Н.Беленький, В.А. Яровенко, и др. // Весник Астраханского государственного технического университета. Серия: морская техника и технология. - 2015. № 2. - С. $31-41$. 3. Пат. 2246103 Российская Федерация, МПК7 G 01 M 15/00. Способ диагностирования двигателей внутреннего сгорания [Текст] / Носырев Д.Я., Краснов В.А.; заявитель и патентообладатель Сам ГАПС. - №2003119976/06; заявл. 01.07.03; опубл. 27.12.2004. 4. Коррекция индикаторной диаграммы рабочего прочесса дизеля методом иифровой фильтрачии на основе преобразований Фурье [Текст]/ А. И. Трунов // Ученые заметки ТОГУ Тихоокеанский государственный университет, Хабаровск - 2016. - Том 7, № 1, - С. 190 200.

\section{Bibliography (transliterated):}

1. Kon'kov,A.U., Lashko, V.A. (2014), Diagnosing of a diesel on the basis of identification of working processes : monograph [Diagnostirovanie dizelja na osnove identifikacii rabochikh processov : monogr.], Dal'nauka, Vladivostok, 356 p. 2. Varbanec, R.A., Belen'kij, P.N., Jarovenko, V.A. (2015), Definition of main specifications of a working process and results of diagnostics of propulsion diesels of motorship " GREISFSWALD ",Vesnik the Astrakhan state engineering university. A series: marine technics\{technical equipment\} and technique, [Opredelenie osnovnykh parametrov rabochego processa I rezul'taty diagnostiki glavnykh dizelej teplokhoda «GREIFSWALD»], №2, pp. 31-41.

3. Nosyrev D.J., Krasnov V.A. (2004), Way of diagnosing of explosion engine: pat. 2246103 Russian Federation, MPK7 G 01 M 15/00 [Sposob diagnostirovanij dvigateley vnutrennego sgoranija] The applicant and patentoobladatel Itself ГАПC. №2003119976/06; zajavl.. 01.07.03; opubl.. 27.12.2004. 4.Trunov, A.I. (2016), Correction of the display glow iris of a working process of a diesel by a method of a digital seepage on the basis of Fourier transforms, Scientific notices the TOGA Pacific state university, Khabarovsk, [Korrekcija indikatornoj diagrammy rabochego processa dizelja metodom cifrovoj fil'tracii na osnove preobrazovanij Fur'e], Tom 7, № 1, pp. 190-200/

Поступила в редакичию 05.10.2017 г.

Яковенко Анатолий Юрьевич - соискатель ученой степени канд. техн. наук при кафедре СЭУ НУ «ОМА», технический директор ДП (дочернее предприятие) «Интреско ЛТД», Одесса, Украина, e-mail: delta0090@ gmail.com, моб. тел. +380503168068 .

\section{МЕТРОЛОГІЧНІ ХАРАКТЕРИСТИКИ РЕЄСТРАЦІЇ ВИСОКОГО ТИСКУ В СИСТЕМАХ ПАЛИВОПОДАЧІ ДИЗЕЛІВ ІЗ ЗАСТОСУВАННЯМ КОМБІНОВАНОГО ДАТЧИКА}

\section{А.Ю. Яковенко}

Представлено методику гармонійного аналізу метрологічних характеристик запису нестаціонарних процесів. Використано метод Фур'є в додатку до завдань обробки даних у графічній і табличній формі. Коефіцієнти Фур'є обчислені за допомогою наближеного методу інтегрування з використанням формул прямокутників. Розроблено алгоритм і програма, адаптовані до виміру параметрів паливоподачі дизелів. Програмна реалізація має широкий спектр опцій, що забезпечує оптимальний інтерфейс і форму подання результатів аналізу. Проведено дослідження амплітудно-частотних параметрів вимірювальної системи на базі комбінованого перетворювача тиску палива. Як перетворювач використаний конструктивний елемент паливних апаратури суднового середнеобертового дизеля. Тиск палива в ПНВТ записувалося двома датчиками: базовим традиційної конструкції й експєріментальнім. Проведено безмоторні випробування в діапазоні частот обертання, що перекривають експлуатаційні режими двигуна. Експериментальні дані оброблені в середовищі розробленого пакета . Визначено амплітудно-частотні характеристики вимірювальної системи в порівнянні комбінованого й базового перетворювача тиску палива. Зроблено дискретне перетворення Фур'є з виводом результату у вигляді графіка амплітуд спектральних складових на екрані і для візуального контролю зворотне перетворення Фур'є також з виводом графіка функції на екран. Встановлено, що система 3 комбінованим перетворювачем має параметри близькі до базового варіанта на всіх режимах за винятком дробового упорскування.

\section{METROLOGICAL CHARACTERISTICS OF THE HIGH PRESSURE REGISTRATION IN INJECTION FUEL SYSTEMS OF DIESEL ENGINES WITH APPLICATION OF THE COMBINED TRANSDUCER}

\section{A.J. Jakovenko}

The technique of the harmonious analysis of metrological characteristics for record of non-stationary processes is presented. Furies method is used in the apply to tasks of graphic and tabulated form data processing. Furies coefficients were calculated by means of the approached integrations method with use of rectangular formulas. The algorithm and the program, adopted for measurement of fuel injection parameters of diesel engines, is developed. Program realization has the wide spectrum of options providing the optimum interface and the form of representation of results of the analysis. It was carried out research of amplitude -frequency parameters of measuring system on the basis of the combined converter for fuel pressure. As the converter was used the constructive element of the marine middle speed diesel fuel injection system. Pressure of fuel in FPHP was measured with two transducers: base of traditional design and experimental. Engineless tests in boards of the of rotation frequencies, overlapping operational limits of the engine, are lead. Experimental data are processed in the environment of the developed package. Peak-frequency characteristics of measuring system in comparison of the combined and base converter of pressure of fuel are certain. Discrete Furies transformation was made with show of result in the form of the diagram of amplitudes the spectral components on the screen and for the visual control return Furies transformation also with a show of the diagram of function to the screen. It is established, that the system with the combined converter has parameters close to a base variant on all modes except the fractional injection. 